\title{
Effect of perceptual cohesiveness on pattern recoding in the block design task
}

\author{
FRED L. ROYER and KENNETH E. WEITZEL \\ Veterans Administration Hospital (Brecksville Division), Cleveland, Ohio 44106
}

\begin{abstract}
In the block design task, one type of intelligence test task, the subject reproduces a square-field pattern by assembling cubes having solid and diagonally divided surfaces. The task requires that the subject recode the perceived stimulus pattern into the alternatives available on the block surface. This experiment examined the effect on response or reproduction time of perceptual cohesiveness of pattern, quantified as the number of adjacent same-colored edges of cubes which abutt to form the pattern. This stimulus variable interacts with the type of design (quantified as total set size for designs constructed of all solid surfaces, all diagonally divided surfaces, or mixtures of the two types of surfaces) and with cuing (presence or absence on stimulus pattern of a superimposed grid congruent with block edges of the constructed design). Cohesiveness increases the difficulty of "mentally slicing" or recoding the pattern into the set of block alternatives. The psychological processes involved in recoding probably are the most important ones underlying the intelligence test task.
\end{abstract}

The block design task has been examined experimentally as an information processing task (Royer, in press). The task has a long history of use in intelligence testing and the fact that performance is determined to a large extent by informational variables suggests that the information processing operations are basic intellectual processes which vary in speed, efficiency, or capacity among individuals. This experiment examines an important perceptual variable that affects the information processing operations in the task.

The most frequently used form of the task is a derivative of the original Kohs (1923) test used in the Wechsler intelligence scales (Matarazzo, 1972). The Wechsler task is classified as a performance test, i.e., a nonverbal one. The task is especially interesting because it correlates well with Full Scale Intelligence Quotient and because it correlates better with verbal scales than some of them do with each other (Matarazzo, 1972, pp. 212-214, 236-237). Also, it is clinically valuable in appraising brain damage (Royer \& Holland, 1975). A task with these characteristics ought to be tapping some very broad, powerful, fundamental, cognitive activities. The evidence suggests that certain basic perceptual organizational properties of the stimulus (pattern goodness and cohesiveness) largely determine task

This work was performed as part of VA Research Project 7600-04. The authors are grateful for the assistance provided by Gary Zychowski in collecting the data, and acknowledge with gratitude the use of space provided by Douglas Detterman of Case Western Reserve University, where the senior author is a Lecturer and Associate in the Department of Psychology. Requests for reprints should be sent to Fred L. Royer, PhD, VA Hospital, Psychophysiological Research Laboratory (Brecksville Division), 10701 East Boulevard, Cleveland, Ohio 44106. difficulty. Thus, we expect that the task demands the linking together of what should be simple but important perceptual and cognitive processes. Task difficulty is what discriminates among various individuals' abilities to do this task, obviously; but it apparently also discriminates among their abilities to do other tasks in which the role of perceptual processes does not seem to be so obvious. Clarification of the role of stimulus parameters in task difficulty should provide clues to both the underlying perceptual processes and the nature of what ought to be a fairly general problem solver.

In the task, the subject is shown a square multicolored pattern and is asked to reproduce it on the top surfaces of multicolored cubes. In the Wechsler Adult Intelligence Scale (WAIS) version, the patterns to be constructed by reproduction are red and white; each block has two surfaces that are solid red, two that are solid white, and two that are diagonally divided half-red and half-white. Some designs are constructed using four blocks, some using nine blocks. The task therefore requires the subject to process a visual pattern, to recode the pattern into units which correspond to the set of alternatives available on the surface of the cubes from which he must construct the design, to make a size transformation of the pattern (design is smaller than block pattern), and to compare his production with the pattern. Success in these operations appears to be related to intelligence measured in a variety of quite different verbal and nonverbal tasks.

In examining the WAIS task as an information processing one, Royer (in press) conjectured that since the task involves the reproduction of visual patterns, the principles of pattern perception should apply to some aspects of performance. The task is 
basically a reproduction one, memory demands are minimal, pattern discrimination is an element only when the observer is comparing his construction with the presented design, and the blocks are randomized before each presentation of a new pattern. Therefore, the principles that apply to the perception of single patterns would be most appropriate. However, with memory and discrimination demands being minimal, a question that needed to be answered was what processing operations might be required by the task that would be affected differentially by parameters of the single stimulus. Royer conjectured that after the initial perceptual coding of the stimulus, the stimulus must be recoded into the alternatives provided by the block surfaces. Much of the time required to do the task would be the sum of the time required to do the initial coding and the subsequent coding.

To examine the recoding of the stimulus pattern into the alternatives provided by the block surfaces, Royer presented one group with a grid superimposed over the stimulus pattern so that the block position was cued. The cued stimulus. was expected to minimize the recoding operation and provided a reference condition for evaluating the role of stimulus structure variables in the task.

The first stimulus parameter which was chosen for the design of stimuli used in the task was total set size. There were two reasons for this: (a) total set size, logarithmically expressed as uncertainty of total set size, provides a measure of the number of successive binary choices of surface-type alternatives; and (b) the perceived redundancy of the single pattern, according to Garner's theory $(1962,1974)$, is determined by the size of the total set of stimuli that can be generated from the given stimulus dimensions and the size of the subset of related stimuli which could be presented from the total set. A number of stimulus patterns were selected from total sets of different sizes, where the total set is defined by all of the possible patterns which could be constructed from a particular set of alternative block faces. For example, a total of 16 configurations can be constructed using four solid red or white surfaces; a total of 256 configurations can be constructed using four diagonally divided surfaces, since each block has four alternative orientations. Total sets with sizes between these values can be constructed using various numbers of solid and diagonally divided blocks.

Royer (in press) found that performance time was a linear function of the uncertainty of total set size for the cued group. For the uncued group whose patterns did not have the superimposed grid, performance time had significant linear and quadratic components. Performance was fairly linear until about 9 bits, at which point there was a marked curvilinear acceleration in the curve. The patterns producing this performance were very much like those which would contribute to differentiating individuals in the intellectual range of most college students.

The second stimulus parameter was pattern redundancy. For the examination of the role of pattern redundancy, Royer also used patterns varying in size of rotation and reflection equivalence set (Garner \& Clement, 1963). Clement and Varnadoe (1967) and Royer (1966) have shown that this variable plays a role in discriminative performance. In the nine block solid designs, analogous patterns of the Garner and Clement (1963) five-dot designs were developed which used a red block where a dot occurred and a white block where a blank space occurred in their designs. Performance in both the cued and uncued groups was a linear function of the uncertainty of the rotation and reflection equivalence set. Designs of the other total set sizes contained patterns of various equivalence set sizes. These designs, particularly where diagonal patterns were present, did not seem to be ordered in any systematic way with respect to equivalence set size. Rather, it appeared, after examining these results, that pattern cohesiveness was a variable that might be more of a determinant of response time than equivalence set size.

An examination of Kohs' (1923) findings seemed to support the possibility that his empirically established hierarchy of design changes which increased the difficulty level could be accounted for fairly completely by the principles of uncertainty of total set size, uncertainty of equivalence set size, and perceptual cohesiveness. His stages for increasing task difficulty were (Kohs, 1923, p. 65):

" 1 . By the use of full colors;

2. By the use of few diagonaled sides;

3. By the use of all diagonaled sides;

4. By turning the design on one of its corners;

5. By eliminating the outside boundary line;

6. By increasing the number of blocks to be used;

7. By increasing dissymmetry in design;

8. By increasing the number of different colors used in each design."

It will be seen that Steps 1, 2, 3, and 6 increase the total set size. Step 7 increases the rotation and reflection equivalence set size which is related to dissymetry (Prokhovnik, 1959). Steps 5 and 8 increase the perceptual cohesiveness of the pattern. Step 4 is a special problem not investigated in these experiments. Step 4 is an operation related to the "oblique effect," reviewed by Appelle (1972). The use of the diagonaled surfaces which tend to generate designs with oblique elements is sufficient for initial investigations of the task. One additional note is that Kohs used blocks and designs with four 
colors rather than two; this gives Step 8 a wider range of alternatives than would be the case with the WAIS blocks. The empirical progression suggests that interactions of the stimulus variables of total set size, equivalence set size, and perceptual cohesiveness should be expected.

The Royer (in press) experiment accounts for five of the steps, but does not account for perceptual cohesiveness in information processing terms. It is possible to conceptualize what might be involved in recoding patterns that would make perceptually cohesive designs more difficult to deal with than less cohesive ones. When two blocks are juxtaposed, the edges at the juncture may be the same color or different colors. If the resultant pattern is reproduced without a cue as to where the block edges occur, a contour congruent with the block edges will be present when the edges are different, but no contour congruent with the block edges will be present when those edges are the same color. A subject faced with the task of reconstructing the block arrangement that generated the design would have to make a mental slice through the design if the edges were the same color. Hence, we can quantify the cohesiveness of the design by counting the number of same colored block edges which abutt. This measure would seem to be an appropriate measure of the number of operations the subject would have to perform on the design, or else the number of recoding operations he would have to perform, if we assume that no additional recoding is necessary when contours conform to block edges.

The purpose of this experiment was to examine the perceptual cohesiveness factor in the task where cohesiveness is defined as the adjacency count. In this experiment, all designs were limited to a rotation and reflection equivalence set size of four and were generated using 16 blocks. The larger number of blocks was used to ensure a wide range of adjacency counts, from 0 to 24 .

\section{METHOD}

\section{Subjects}

The subjects were 50 students of Case Western Reserve University who satisfied course requirements for introductory psychology by their participation. They were divided into two groups of 25 subjects each. There were 12 men and 13 women in the cued group, which reproduced designs with a grid, congruent with block edges, superimposed over the pattern; there were 13 men and 12 women in the uncued group, which reproduced designs without the grid.

\section{Stimuli}

Stimuli were generated so as to examine two stimulus variables, (a) adjacency count and (b) uncertainty of total set size, while controlling rotation and reflection equivalence set size at four. Adjacency count varied in steps of four from 0 to 24 , yielding seven levels of this variable: $0,4,8,12,16,20,24$. Uncertainty of total set size was varied by using solid colored surfaces $(8 \mathrm{red}$ and 8 white) of the blocks only (Type S, solids), or $\log _{2} 2^{16}$ possible configurations, or 16 bits; using eight solid colored
( 4 red and 4 white) and eight diagonaled surfaces (Type $M$, mixed), or $\log _{2} 2^{8}+\log _{2} 4^{8}$ possible configurations, or 24 bits; and using only diagonaled surfaces, or $\log _{2} 4^{16}$ possible configurations, or 32 bits (Type $\mathrm{D}$, diagonaled).

Mathematical constraints imposed by both adjacency counts and limitations on block surface alternatives make it impossible to generate patterns for certain categories or to generate more than one pattern in some categories. We could generate no patterns of 0 or 24 adjacency counts that had an equivalence set size of four by using 8 red and 8 white blocks. Only one pattern could be generated with an adjacency count of 20 . Only one pattern' with an equivalence set size of four and with a zero adjacency count could be generated using diagonaled surfaces only. Where more than one pattern could be generated, two were selected for the experiment. The resultant stimulus set consisted of 36 designs. The Type $S$ set had two designs each of adjacency counts $0,4,12,16,20$, and 24 . The Type $D$ set had one design of adjacency count 0 and two designs each of count 4 , $8,12,16,20$, and 24 . Each set of designs was reproduced with and without a superimposed grid demarcating the position of the block edges.

Examples of stimuli of each type and several adjacency counts are shown in Figure 1. The stimuli were presented on cards, $7.8 \times 13 \mathrm{~cm}$; the designs were centered and were $4 \times 4 \mathrm{~cm}$. The blocks used to construct the designs were $2.7-\mathrm{cm}$ cubes.

\section{Design}

The experimental design was a mixed design. The betweensubjects variable was Cuing. Half of the subjects had the cued designs (Group Cued); the other half had the uncued designs (Group Uncued). The within-subjects variables were Adjacency Count and Type (Solid, Mixed, or Diagonaled).

\section{Procedure}

The subjects were scheduled two at a time. Upon arrival, they were assigned to one of two experimenters, Each experimenter alternately tested two subjects in each group.

The appropriate deck of 36 cards was shuffled to randomize the order of the designs.

The subjects were shown two blocks. The experimenter pointed out the different surfaces and demonsirated, by placing the two cubes together, how various solid and diagonally divided surfaces could be juxtaposed. Four different ways of juxtaposing diagonally
음

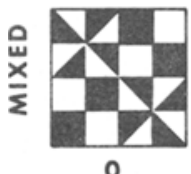

0

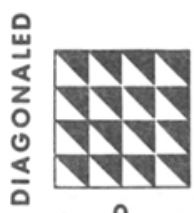

0

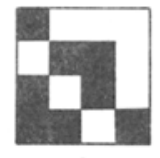

8

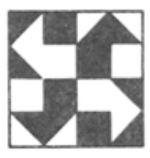

8

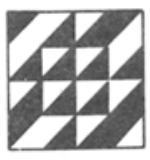

8

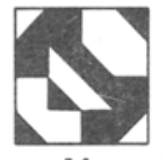

16

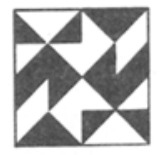

16

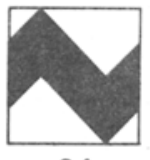

24

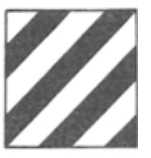

24
Figure 1. Examples of stimuli of each type and of four of the seven adjacency counts. No designs exist for Type $\mathbf{S}$ designs with 0 and 24 adjacency counts. 
divided blocks were demonstrated. It was then explained that designs on the first card could be reproduced with four blocks arranged in a square. The subjects were instructed to say "finished" as soon as they were satisfied with the reproduction. Instructions pointed out that the more time that the subject spent looking at the design when finished, the more his score would be penalized; therefore, he should be as quick as possible while being as accurate as possible. The subjects were told not to touch the blocks until a card was turned over and the experimenter said "go."

The sample problem of a 4-block design, requiring 2 diagonaled and 2 solid surfaced blocks for reproduction, was presented to the subject. When he completed this sample, he was told that he would do the same thing with designs using 16 blocks instead of 4 . The shuffled deck of 36 designs was then presented. Blocks were randomized before each trial by tossing them onto the table.

At the completion of the experiment, its purpose was explained to the subjects and its relationship to problems of perception and psychopathology briefly noted.

\section{RESULTS}

\section{Errors}

The error rate was $1.78 \%$ for Type S designs, $6.51 \%$ for Type $M$, and $7.23 \%$ for Type $D$. There was no systematic distribution of errors according to adjacency count. The error rate is reasonably low and indicates that subjects attempted to be accurate in their performance.

\section{Response Time}

The means of the response times for constructing each pattern are presented in Table 1 together with the coded description of each design.

When there was more than one pattern to a pattern class, the response times (RT) or the log RTs were averaged for each subject for use in the analyses. These scores were examined by analysis of variance. Since there were no patterns of Type $S$ with adjacency counts of 0 or 24 , two analyses were done; one analysis was made of solid, mixed, and diagonal-type patterns with adjacency counts from 4 to 20; the other was made of mixed and diagonal-type patterns with adjacency counts from 0 to 24 .

Means and standard deviations of individual patterns appeared to be proportional, due to skewed distributions. Therefore, analyses of variance were done for both raw and logarithmically transformed response times. The analysis of transformed scores indicated that the variability of the raw score data tended to deflate the $F$ ratios. Only one effect in the two analyses to be reported was not already significant at the probability level of .01 or less in the raw score analysis. Therefore, the results of the analyses of transformed scores will be reported, although the means, which are graphed for illustrative purposes, will be of raw score data.

The analysis of variance of $\log$ response times for the three types of stimuli in adjacency counts of

Table 1

Description of Designs and Mean Construction Time

\begin{tabular}{|c|c|c|c|c|c|c|}
\hline \multicolumn{3}{|c|}{ Solid } & \multicolumn{3}{|c|}{ Mixed } & Diagona \\
\hline Code & Cued & $\begin{array}{l}\text { Un- } \\
\text { cued }\end{array}$ & Code & Cued & $\begin{array}{l}\text { Un- } \\
\text { cued }\end{array}$ & Code Cued \\
\hline
\end{tabular}

Adjacency Count 0

1RWR

443W

W 122

$35.2 \quad 35.0$

RWR3

34WR 3333

21RW $37 . \quad 365 \quad 3333$

RW12 37.1 $36.5 \quad 3333$

$31.3 \quad 29.9$

WR4

3333

Adjacency Count 4

RWWR

WRRW

RWWR

WRRW

WRWR

RWRW

WRRW

RWWR

WWRR

RRWW

WWRR

RRWW

RWWW

WRRW

RWRW

RRWR

WWWR

WRRR

WRWW

RRWR

WWRR

RRWW

RRWW

WWRR

RRRR

WWWR

WWWR

RWWR

RRRW

RWWW

RWWW

RRRW

WWWW

WWWW

RRRR

RRRR
R32R

IRR4

W14W

$33.8 \quad 34.2$

1212

$27.3 \quad 26.3$

WRWR

WRWR

3

212

Adjacency Count 8

2R41

$\begin{array}{lll}18.6 & 19.8 \quad \text { IWRW }\end{array}$

WRW3

36.

$36.2 \quad 39.9$

4444

32R4

RW 12

$25.6 \quad 26.8$

WR 11

WR 22

RW21

$38.1 \quad 42.2$

Adjacency Count 12

IR31

$24.9 \quad 29.6$

WW4R

R2WW

$31 \mathrm{R} 3$

W4W4

$21.5 \quad 20.0$

W4W

W4W4

$34.3 \quad 53.3$

Adjacency Count 16

4RW3 1432

R31W $345 \quad 501 \quad 2421$

$\begin{array}{llll}\text { W13R } & 34.5 & 50.1 & 1312\end{array}$

1WR2

2341

RWRW

22.924 .4

3124

3124
4213

RWRW

$41.4 \quad 58.3$

1432

4144

2232

4123

Adjacency Count 20

$\begin{array}{lllllll}\text { R1WW } & & & 2323 & & \\ \text { R214 } & & & 4242 & & \\ \text { 234R } & 40.4 & 79.9 & 3131 & 53.1 & 75.1 \\ \text { WW3R } & & & 1414 & & \\ \text { R23R } & & & 4114 & & \\ \text { 3142 } & & & 2332 & & \\ \text { W32W } & 36.2 & 50.2 & 4114 & 57.4 & 76.2 \\ \text { RWWR } & & & 2332 & & \\ \end{array}$

\section{$41.3 \quad 44.5$}

344

$44.0 \quad 45.9$

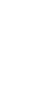


Table 1 Continued

\begin{tabular}{|c|c|c|c|c|c|c|c|c|}
\hline \multicolumn{3}{|c|}{ Solid } & \multicolumn{3}{|c|}{ Mixed } & \multicolumn{3}{|c|}{ Diagonal } \\
\hline Code & Cued & $\begin{array}{l}\text { Un- } \\
\text { cued }\end{array}$ & Code & Cued & $\begin{array}{l}\text { Un- } \\
\text { cued }\end{array}$ & Code & Cued & $\begin{array}{l}\text { Un- } \\
\text { cued }\end{array}$ \\
\hline \multicolumn{9}{|c|}{ Adjacency Count 24} \\
\hline & & & $\begin{array}{l}41 W 3 \\
\text { RR IW } \\
\text { RR2W } \\
32 W 4\end{array}$ & 37.9 & 53.3 & $\begin{array}{l}4242 \\
2424 \\
4242 \\
2424\end{array}$ & 51.3 & 73.3 \\
\hline & & & $\begin{array}{l}41 W W \\
\text { RR } 14 \\
23 R R \\
\text { WW32 }\end{array}$ & 40.3 & 77.8 & $\begin{array}{l}4141 \\
2323 \\
4141 \\
2323\end{array}$ & 53.9 & 89.8 \\
\hline
\end{tabular}

Note-Design code: $R=$ solid red surface, $W=$ solid white surface: $I$ = diagonal: negative gradient, red below diagonal; 2 = diagonal: positive gradient, red above diagonal; 3 = diagonal: negative gradient, red above diagonal; 4 = diagonal: positive gradient, red below diagonal.

4 to 20 indicated that there was a significant difference between the means for all main effects: Cuing, $\mathrm{F}(1,48)=8.41, \mathrm{p}<.01$; Type, $\mathrm{F}(2,96)=825.9$, $\mathrm{p}<.01$; and Adjacency Count, $\mathrm{F}(4,192)=22.22$, $\mathrm{p}<.01$. All interactions were significant: Cuing by Type, $F(2,96)=21.99, \mathrm{p}<.01$; Cuing by Adjacency Count, $\mathrm{F}(4,192)=9.23, \mathrm{p}<.01$; Type by Adjacency Count, $F(8,384)=36.98, p<.01$; and Cuing by Type by Adjacency Count, $F(8,384)=4.42$, $\mathrm{p}<.01$.

The significant three-way interaction is important in explaining the main effects and other interactions. The three-way interaction is graphed in Figure 2. (Points for Type $M$ and Type D with Adjacency Counts of 0 and 24 have been added for purposes of discussion of the second analysis.) It is quite evident that the Type $S$ designs are not influenced either by cuing or adjacency count. Apparently these patterns are easily analyzed, regardless of adjacency count. The total time required to complete the designs may be more a function of the number of blocks to be handled than of the number of analytic operations. The relative proportion of the response time required for the analytic operations may be so small with respect to the time required to assemble the blocks that the effect of cohesiveness is masked. In the previous study of this task (Royer, in press), the mean time for Solid 4-block patterns was $5.81 \mathrm{sec}$ and for Solid 9-block patterns was $11.65 \mathrm{sec}$, while it is 22.65 in the present one. The average response time per block in each case is quite similar, 1.45, 1.29 , and $1.42 \mathrm{sec}$, respectively, and could be primarily a measure of the block selection and motor response component of the task, the remaining time for the processing of stimulus information being lost in the variance of the motor response component.

Before examining this complex interaction further for the effects of Adjacency Count, the results of the analysis of variance of the effects of Adjacency
Count on performance across the full range of adjacency counts of 0 to 24 for the Type $D$ and Type $\mathbf{M}$ designs is presented for purposes of discussing the interaction of Type by Adjacency Count. The analysis of variance of $\log$ scores indicated that there are significant differences among the means for all main effects: Cuing, $F(1,48)=12.71, p<.01$; Type, $F(1,48)=199.4, p<.01 ;$ and Adjacency Count, $F(6,288)=88.77, p<.01$. The interaction of Cuing by Type is not significant, $F(1,48)<1.0$, $p>.05$. The interaction of Cuing by Adjacency Count is significant, $F(6,288)=15.16, p<.01$, as is the interaction of Type and Adjacency, $F(6,288)=$ $25.75, \mathrm{p}<.01$. The three-way interaction of Cuing by Type by Adjacency Count is significant, $\mathrm{F}(6,288)=4.37, \mathrm{p}<.01$. The results are consistent with the analysis including the Type $S$ designs, except for the interaction of Cuing by Type.

Figure 3 presents the data in this analysis for the interaction of Cuing by Adjacency Count. As noted earlier, when the adjacency count is 0 , there is a contour that is congruent with each block edge; one would not expect that the addition of the cuing grid would contribute anything more to the speed with which the mental slicing of the design could be accomplished. Hence, it should take just as much time to do the task in both conditions when the count is 0 . As the adjacency count increases, the number of recoding operations would be expected to increase as well; hence, the performance curves should become more and more divergent, being maximally so when the count is 24 . The performance curves conform generally to the expectation. It is clear that adjacency count, as a measure of the perceptual cohesiveness of the design, is an important variable in the block design task, one that systematically

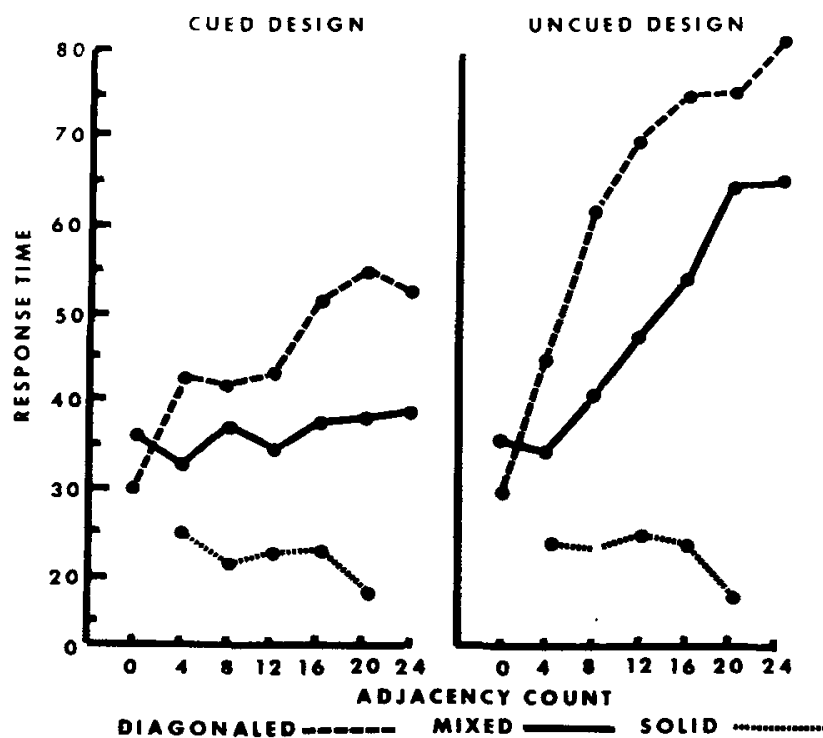

Figure 2. Respnse or construction lime in seconds as a function of cuing, type, and adjacency count. 


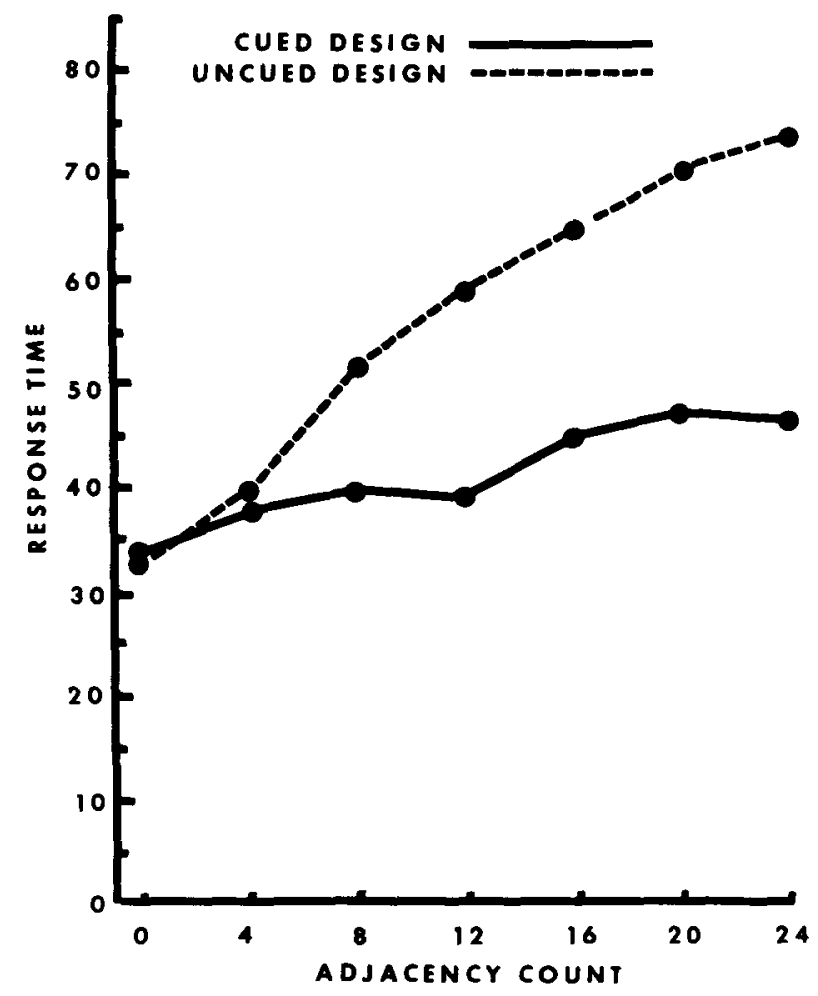

Figure 3. Response or construction time in seconds as a function of cuing and adjacency count for the Type $M$ and $D$ designs.

contributes to performance time.

The strength of the effect is further evident in the increasing response time attributable to adjacency count for the Cued group. One might expect that with cuing provided, the response curves for the mixed and diagonaled designs would be similar to that of the Type $S$ designs. That is, the cuing might be expected to eliminate any effect that the adjacency count (the perceptual cohesiveness) would have on the response time. Returning to the complex interaction and Figure 2, it can be seen that the data show a differential rate of increasing response time for increasing adjacency count in the curves of the Cued group, although the differential rate is considerably less than that of the Uncued group. This means that, even with cuing provided, the perceptual cohesiveness affects the time required to recode the pattern into the alternatives available on the block surfaces.

\section{DISCUSSION}

\section{Adjacency Count and Cuing}

Since perceptual cohesiveness (measured by adjacency count) affects the recoding time even when cuing is provided, we must assume that there is an additional operation required in the recoding process when there is no cuing provided. Since the additional operation is related to the number of adjacencies in the pattern, the time required will increase multiplicatively with respect to the time required when cuing is provided. We also must assume that the operation involves diagonal surfaces since the type of design also interacts. The main difference between the Type $D$ and Type $M$ designs is that Type $M$ contains 8 while Type D contains 16 diagonaled surfaces; Type $\mathrm{S}$ designs have no diagonaled surfaces and performance is unaffected by adjacency count of the design. The differential slopes of the performance curves for the Type $\mathbf{M}$ and Type $\mathrm{D}$ designs for both the Cued and Uncued groups vary in a way that supports this interpretation, which is, roughly speaking: the greater the number of diagonaled surfaces, the greater the time required as adjacencies increase; and for a given number of diagonaled surfaces, absence of cuing increases the time required as the adjacencies increase, but at a considerably greater rate than when cuing is present.

There is a possible explanation for these results. Among the obvious operations in the sequence of breaking up the pattern, selecting a type of surface, rotating the surface, rechecking orientations of the surface, making the appropriate size transformations of presented and constructed pattern, may be one that requires not only the mental checking of the orientation of the particular diagonaled surface, but the mental checking of the orientation of the adjacent block to determine whether the resultant juxtaposition will produce the proper cohesive configuration. The more cohesive the pattern, the greater the number of checks which have to be made of same color adjacencies. While this check may have to be done in the Cued condition to satisfy the criterion that a proper pattern is being presented, the mental slicing operation and the check of an adjacent block may have to be repeated, thereby adding more time, and adding it for each adjacency count. Another possibility is that no size transformation is required with cued designs but is with uncued designs since this would be a way to ensure that the mental slice had been made at the appropriate point. The Goldstein-Scheerer Cubes Test, which is a variation of the Kohs test and which is used in testing for brain damage, makes use of size transformation as well as cuing in varying the difficulty levels of the task.

\section{Other Variables}

There are two cases which seem to be discrepant with the general trend of the data. One of these, as indicated earlier, is the single design of Type $S$ which can be constructed with an adjacency count of 20 . This design consists of a band of red and a band of white color. Most subjects approached this design differently from others; they seemed to be able to "chunk" the parts of the design, quickly assembling a group of several blocks of a single color together, then another group of a different color to form the design. The other design is the single Type $\mathrm{D}$ design with an adjacency count of 0 ; this design has all 
16 blocks oriented in the same way. This repetitious pattern was completed much more rapidly than the Type $M$ designs of the same adjacency count. Both designs have properties that are not characteristic of the other designs, and the results suggest that still other variables may operate in certain circumstances. For example, the repetition of a small, perceptually distinct subunit of a pattern may provide a type of informational redundancy (repetition redundancy) different from that present in other designs, thereby speeding up the processing operations or bypassing other operations that are required for dealing with the other patterns. Also, some designs may be degenerative. That is, the pattern duplicates one of a very few patterns which can be constructed with fewer blocks and therefore requires no more processing time than is needed for constructing the pattern with fewer blocks, only as much extra time as is needed to manipulate more blocks.

The results of this study and of Royer (in press) make it possible to identify most of Kohs' hierarchical stages of task difficulty as manipulations of several informational variables: $\log _{2}$ total set size, $\log _{2}$ rotation and reflection equivalence set size, and adjacency count. The interaction of the variables accounts for Kohs' ordering. The one step that has not been investigated is the effect of "turning the design on one of its corners," which is a $45^{\circ}$ rotation. We believe the present results and our interpretation of them suggest an explanation and the sort of results that might be expected in further research on this problem. The $45^{\circ}$ rotation tends to create diagonal contours on the perimeter and to reorient the diagonal contours of the blocks in vertical and horizontal directions. As discussed earlier, there is reason to believe that the interaction of adjacency count with type (total set size) may involve operations for analyzing or recoding the patterns having diagonal contours so that they conform to the alternatives available on the block surfaces. In the usual method of presentation of the stimuli, as used here, the orientation of the blocks and pattern perimeter is rectilinear. The diagonal contours are essentially incongruent with this orientation. If Kohs is correct, forcing the use of the blocks in an orientation rotated $45^{\circ}$ should require more recoding operations than needed to process the patterns using rectilinear orientation of the blocks. Our examination of the particular patterns used by Kohs indicates that all are symmetrical on one diagonal axis, hence, do create a horizontal orientation to the diagonaled surfaces. Other patterns could be generated, however, with symmetry on the horizontal or vertical axis in a rectilinear orientation; such patterns would not produce the same type of horizontally organized Gestalt under $45^{\circ}$ rotation. It is quite possible that such patterns would be less difficult than are the patterns symmetrical on the diagonal axis when rotated $45^{\circ}$. It seems more parsimonious, lacking additional evidence, to assume that the difficulty lies in the relative incongruity of perimeter orientation and contour orientation and consequent effects on the pattern recoding operation.

\section{Perceptual Recoding and Intelligence}

The results show that the adjacency count of similar colored surfaces of the blocks used to construct the stimulus pattern is a measure of a stimulus property that affects performance. That property is perceptual cohesiveness. ${ }^{2}$ A cohesive pattern seems to be more difficult to analyze into subunits which are the alternatives provided by the block surfaces. We have chosen to describe this analytic process as a recoding one and conclude that cohesive patterns are more difficult to recode than noncohesive ones. We assume that the stimulus is coded by the nervous system into its own representation. That code is then examined for possible matches with the coded representation of the various block surfaces stored in memory. The accepted matches provide a recoded representation which is, in turn, used as a model for generating the construction. The least cohesive patterns may be thought of as a string of code elements that is easily segmented according to a rule that contours are boundaries of code segments. The most cohesive patterns lack contours at points which conform to block surfaces or have contours that suggest conformation until the actual construction disconfirms the possibility. These patterns may be thought of as a string of code elements that is difficult to segment because many elements of the string have to be compared to find a multiple set of alternatives that will match a larger portion of the string.

This interpretation of the task's required psychological processes suggests, of course, that pattern perception may be studied with this or similar tasks in which the number and types of alternatives are varied. But a more far reaching possibility is that whatever the recoding operation may be, it is highly general and is so fundamental in cognitive activity that it is an index of intelligence.

There are at least two approaches to explaining how the recoding process required in this task could be related to various measures of intelligence. One approach is to consider a general problem solver which is required in this and other tasks. Another approach is to consider a processor that has limited capacity or limited speed, or both.

A general problem solver might have as one of its important functions the analysis of a complex code by comparing a set of stored smaller code units. Any rules which have been learned for dealing with the smaller code units can then be applied to the more complex code. Such a problem solver could be highly general if the system used only its own internal code. Like a computer, all input, regardless of the nature of the source device, is coded into a standard which 
is used throughout the system. Thus, regardless of modality or type of original input information, the internal representation is subject to analysis providing some set of alternatives are available. The block design task measures the speed with which one is able to analyze a two-dimensional visual pattern structure in terms of the arbitrary alternatives of a completely different three-dimensional system. Perhaps this is a clue to the nature of the process and of the General Problem Solver. When we construct models or analogies, we analyze one system in terms of the arbitrary alternatives of a different one. To the extent that matches are found, we term the models or analogies meaningful. The General Problem Solver, containing a set of learned or built-in rules for matching codes could be expected to function with perceptual, verbal, associative, or other stored representations with equal facility. At the perceptual level, some rule restrictions might limit the analysis to the use of modality-specific alternatives. The verbal-associative representations might permit violation of such rules by linking codes cross-modally. The block design task would be a simple test of the efficiency of the solver with a particular limited type of code. Task performance might be expected to be correlated with performance on other tasks of a very different type in which the problem solver participated. What the analog of perceptual cohesiveness might be for psychological domains other than visual pattern analysis is difficult to anticipate, although we believe that run structure of auditory patterns studied by Royer and Garner (1966) provide possible analogs: The symmetry and syntely measures developed by Psotka (1975) for binary pattern structure are excellent possibilities for a cohesiveness analog.

The other approach is a visual information processor that shares memory and speed limitations with other information processors. It may be that the number and type of blocks used (total set size) and the number of items of code to be examined increase the amount of information to be held in memory; thus, the task might be measuring a kind of channel or memory capacity shared commonly with other processors. Perhaps the speed of central nervous system activity, which is to say processing time, differs from one individual to another for some standard number of operations required to process a stimulus with particular properties. The task, by analogy to computers, would measure cycle-time. Speed limitations in a processing might not only increase the time for the processing directly but indirectly as well. Temporal decay characteristics of iconic memory may increase the number of data reentries necessary to complete the task. The processing of information other than visual information might be affected by the same limitations and produce a structure of intercorrelations like those found in the Wechsler.

The explanations in terms of capacity and speed of operating are often implicit, if not explicit, in theories of intelligence, but they have never been substantiated experimentally. The hypothesized General Problem Solver is therefore as reasonable an explanation as those of capacity and speed limitations of the system. The two approaches are not mutually exclusive. Not only the rules-repertoire, but required memory capacity, decay time in iconic and short-term memory, as well as execution time might all affect efficiency of a general problem solving system.

\section{REFERENCES}

Apelle, S. Perception and discrimination as a function of stimulus orientation: The "oblique effect" in man and animals. Psychological Bulletin. 1972, 78. 266-278.

Clement, D. E..\& Varnadoe. K. W. Pattern uncertainty and the discrimination of visual patterns. Perception \& Psychophysics. 1967, 2, 427-431.

GARNER. W. R. Uncertainty and structure as psychologicul con cepts. New York: Wiley, 1962

Garner. W. R., \& Chement, D. E. Goodness of paltern and pattern uncertainty. Joumal of Verbal Leaming and Verbal Behavior. 1963, 2. 446-452.

GARNER, W. R. The processing of intormation and structure. New York: Halsted Press, 1974.

Kонs, S. C. Intelligence measurement. New York: Macmillan. 1923.

Matarazzo, J. D. Wechsler's measurement and appraisal of adult intelligence. Baltimore: Williams and Wilkins. 1972.

Prokhovnik. S. J. Pattern variants on a square tield. Psychometrika. 1959. 24, 329-341.

Psotкa, J. Simplicity. symmetry and syntely: Stimulus measures of binary pattern structure. Memon \& Cognition, 1975, 3. 434-444.

Royer, F. L. Figural goodness and internal structure in perceptual discrimination. Perception \& Psychophysics, 1966. 1. 311-314.

ROYER, F. L. Information processing in the block design task. Intelligence, in press.

Royer, F. L., \& GARNer, W. R. Response uncertainty and perceptual difficulty of auditory temporal patterns. Perception \& Psychophysics, 1966, 1, 41-47.

ROYER, F. L., \& Holland. T. R. Rotational transformation of visual figures as a clinical phenomenon. Psychological Bulletin. $1975,82.843-868$.

\section{NOTES}

1. Subsequent explorations of the application of the Prokhovnik (1959) approach to patterns composed of bivariate dimensions to patterns composed of multivariate dimensions show that other such patterns exist.

2. Perceptual cohesiveness is defined and quantified in this study in terms of the task demands. We would expect that stimulus scaling by a purely perceptual judgment would produce an ordering similar to that resulting from the adjacency count.

(Received for publication June 14, 1976; revision accepted October $27,1976$. ) 